\title{
UNIFORM ASYMPTOTIC ESTIMATES OF HYPERGEOMETRIC FUNCTIONS APPEARING IN POTENTIAL THEORY
}

\section{Domingo Pestana and José M. Rodríguez}

\section{Introduction}

In [5], G. B. Folland obtained an expansion in spherical harmonics of the Poisson-Szegö kernel for the unit ball $\mathcal{B}$ in $\mathbf{C}^{n}$ given by

$$
\mathcal{P}_{n}(z, w)=\frac{1}{\omega_{2 n}} \frac{\left(1-|z|^{2}\right)^{n}}{|1-\langle z, w\rangle|^{2 n}}, \quad z \in \mathcal{B}, w \in \partial \mathcal{B}
$$

where $\langle z, w\rangle$ denotes the standard scalar product in $\mathbf{C}^{n}$

$$
\langle z, w\rangle=z_{1} \bar{w}_{1}+\cdots+z_{n} \bar{w}_{n}
$$

and $\omega_{2 n}$ is the $(2 n-1)$-dimensional Lebesgue measure of the unit sphere of $\mathbf{C}^{n}$.

Let $\Delta_{\mathcal{B}}$ denote the Laplace-Beltrami operator associated with the Bergman metric on $\mathcal{B}$,

$$
\Delta_{\mathcal{B}}=\frac{4}{n+1}\left(1-|z|^{2}\right) \sum_{i, j=1}^{n}\left(\delta_{i j}-z_{i} \bar{z}_{j}\right) \frac{\partial^{2}}{\partial z_{i} \partial \bar{z}_{j}} .
$$

This is the basic invariant differential operator on the symmetric space $S U(n, 1) / U(n)$ $\approx \mathcal{B}$. The solution of the Dirichlet problem

$$
\begin{aligned}
\Delta_{\mathcal{B}} u=0 & \text { in } \mathcal{B}, \\
u=f & \text { in } \partial \mathcal{B},
\end{aligned}
$$

with continuous boundary data $f$ is given by the representation formula

$$
u(z)=\int_{\partial \mathcal{B}} \mathcal{P}_{n}(z, w) f(w) d w .
$$

If $\mathcal{H}_{n}^{p, q}$ denotes the linear space of restrictions to $\partial \mathcal{B}$ of harmonic polynomials $g(z, \bar{z})$ on $\mathbf{C}^{n}$ which are homogeneous of degree $p$ in $z$ and degree $q$ in $\bar{z}$, the solution of the Dirichlet problem (1.1), with $f \in \mathcal{H}_{n}^{p, q}$, is given by

$$
u(r \eta)=S_{n}^{p, q}(r) f(\eta), \quad 0 \leq r \leq 1, \eta \in \partial \mathcal{B},
$$

where

$$
S_{n}^{p, q}(r)=r^{p+q} \frac{F\left(p, q ; p+q+n ; r^{2}\right)}{F(p, q ; p+q+n ; 1)} .
$$

By $F(a, b ; c ; t)$ we denote the usual Gauss hypergeometric function

$$
F(a, b ; c ; t)=\sum_{k=0}^{\infty} \frac{(a)_{k}(b)_{k}}{(c)_{k}} \frac{t^{k}}{k !}
$$

Received May 18, 1995, revised November 2, 1995.

1991 Mathematics Subject Classification. 33C05, 33C55, 31B15.

Key words and phrases: hypergeometric functions, spherical harmonics, higher dimensional capacity. 
where $(u)_{k}$ is the Pochhammer symbol

$$
(u)_{k}=u(u+1) \cdots(u+k-1)=\frac{\Gamma(u+k)}{\Gamma(u)} .
$$

The formula (1.2) points to the crucial role of $S_{n}^{p, q}$ in the expansion of the PoissonSzegö kernel in spherical harmonics. In fact,

$$
\mathcal{P}_{n}(r \eta, w)=\sum_{p, q=0}^{\infty} S_{n}^{p, q}(r) H_{n}^{p, q}(\langle\eta, w\rangle)
$$

where $H_{n}^{p, q}(\langle\cdot, w\rangle) \in \mathcal{H}_{n}^{p, q}$ is the zonal harmonic with pole $w$, cf. [5].

If one wants to use the expansion in spherical harmonics, then one is required to know uniform estimates of $F(p, q ; p+q+n ; t)$ in the variable $t$ when the parameters $p, q$ grow, in order to obtain bounds of integrals involving $S_{n}^{p, q}$ (see e.g., Theorem 2 below). For $q=p+a$ with $a$ bounded, Watson [9] [6, p. 237] gave the asymptotic behavior of such an $F$. However, we will need more general estimates.

In this paper, we study the asymptotic behaviour of

$$
F(q, m q ; q+m q+n ; t),
$$

and we obtain the following uniform estimate where $B(\cdot, \cdot)$ denotes the Beta function.

Theorem 1. There exists a universal constant $C$, not depending on $n, p, u, m, z$, such that, for all real numbers, $u, p \geq 0, m, n \geq 1,0 \leq z<1$, if we denote

$$
G=F(p+u, m p+1 ;(m+1) p+u+n+1 ; z) B(m p+1, p+u+n),
$$

then

$$
G \geq C L
$$

where

$$
L=t_{0}^{m p+1}\left(1-m\left(1-t_{0}\right)\right)^{p+u}\left(1-t_{0}\right)^{n-1}\left(\frac{1-z}{a^{2}-b^{2} z}\right)^{1 / 4} \frac{1}{m \sqrt{p+1}}
$$

and

$$
\begin{gathered}
t_{0}=\frac{a+b z-\sqrt{(1-z)\left(a^{2}-b^{2} z\right)}}{2 z}=\frac{2}{a+b z+\sqrt{(1-z)\left(a^{2}-b^{2} z\right)}}, \\
a=1+\frac{1}{m}, \quad b=1-\frac{1}{m} .
\end{gathered}
$$

Also, this result is sharp in the sense that

$$
\lim _{p \rightarrow \infty} \frac{G}{L}=\sqrt{2 \pi} .
$$

By making the choices $u=1 / m, p+u=q$, we have

Corollary. There exists a universal constant $C$, not depending on $n, q, m, z$, such that, for all real numbers, $m, n \geq 1, q \geq 1 / m, 0 \leq z<1$, if we denote

$$
G=F(q, m q ; q+m q+n ; z) B(m q, q+n),
$$

then

$$
G \geq C L
$$

where

$$
L=t_{0}^{m q}\left(1-m\left(1-t_{0}\right)\right)^{q}\left(1-t_{0}\right)^{n-1}\left(\frac{1-z}{a^{2}-b^{2} z}\right)^{1 / 4} \frac{1}{m \sqrt{q+1}}
$$


Observe that without loss of generality, we can suppose $m \geq 1$ because of the symmetry of the hypergeometric function in the two first parameters. It is not possible to obtain a similar uniform upper bound of $F$ because $L$ is zero for $z=1$. However, usually the hard inequalities involve lower bounds. One might think that the hypothesis $p=m q$ is too restrictive, but this is enough to prove some results in which $p$ and $q$ grow independently (see Theorem 2 below). On the other hand, Theorem 2 is sharp.

This uniform estimation of $S_{n}^{p, q}$ allows us to obtain an integral expression for the $\alpha$ energy of a complex measure supported in $\partial \mathcal{B}$. We recall that the $\alpha$-energy is defined by

$$
J_{\alpha}(\mu)=\iint_{\partial \mathcal{B} \times \partial \mathcal{B}} \Phi_{\alpha}(d(x, y)) d \bar{\mu}(x) d \mu(y)
$$

where

$$
\Phi_{\alpha}(t)= \begin{cases}\log \frac{1}{t}, & \text { if } \alpha=0, \\ \frac{1}{t^{\alpha}}, & \text { if } 0<\alpha<2 n,\end{cases}
$$

and $d(x, y)$ is a distance in $\partial \mathcal{B}$.

More concretely, we have the result

Theorem A. [4] If $\mu$ is a complex measure supported on $\partial \mathcal{B}$ and $d(z, w)=$ $|1-\langle z, w\rangle|^{1 / 2}$, we have for $0<\alpha<2 n$ that

$$
J_{\alpha}(\mu) \asymp \int_{0}^{1}\left[\int_{\partial \mathcal{B}}\left|\mathcal{P}_{\mu}(r \xi)\right|^{2} d \xi\right] r^{\alpha / 2-1}\left(1-r^{2}\right)^{n-\alpha / 2-1} d r
$$

where $\asymp$ means that the ratio of the left side to the right side is between two constants which can depend on $n$ and $\alpha$, and $\mathcal{P}_{\mu}$ denotes the invariant Poisson extension of $\mu$, which we recall is defined by

$$
\mathcal{P}_{\mu}(z)=\int_{\partial \mathcal{B}} \mathcal{P}_{n}(z, w) d \mu(w), \quad z \in \mathcal{B}
$$

Theorem $\mathrm{A}$ is one of the keys to obtain a capacity distortion result [4] under inner functions. Recall that if $E$ is a closed subset of $\partial \mathcal{B}$, then

$$
\left(\operatorname{cap}_{\alpha}(E)\right)^{-1}=\inf \left\{J_{\alpha}(\mu): \mu \text { a probability measure supported on } E\right\} .
$$

Recall also that an inner function is a bounded holomorphic function from the unit ball $\mathcal{B}$ of $\mathbf{C}^{n}$ into the unit disk $\Delta$ of the complex plane such that the radial boundary values have modulus 1 almost everywhere. If $E$ is a non-empty Borel subset of $\partial \Delta$, then we let $f^{-1}(E)$ denote the subset of $\partial \mathcal{B}$,

$$
f^{-1}(E)=\left\{\xi \in \partial \mathcal{B}: \lim _{r \rightarrow 1} f(r \xi) \text { exists and belongs to } E\right\} .
$$

Theorem B. [4] If $f$ is inner in the unit ball of $\mathbf{C}^{n}, f(0)=0$, and $E$ is a Borel subset of $\partial \Delta$, then we have:

(i) If $0<\alpha<2$ (and also $\alpha=0$ if $n=1$ ), then

$$
\operatorname{cap}_{2 n-2+\alpha}\left(f^{-1}(E)\right) \geq C(n, \alpha) \operatorname{cap}_{\alpha}(E) \text {. }
$$

(ii) If $\alpha=0$ and $n \geq 2$, then

$$
\frac{1}{\operatorname{cap}_{2 n-2}\left(f^{-1}(E)\right)} \leq C(n)\left(1+\log \frac{1}{\operatorname{cap}_{0}(E)}\right) \text {. }
$$


Corollary. With the same hypotheses of Theorem B, we have

$$
\operatorname{Dim}\left(f^{-1}(E)\right) \geq \operatorname{Dim}(E)+2 n-2
$$

where Dim denotes Hausdorff dimension with respect to the distance $d(z, w)=$ $|1-\langle z, w\rangle|^{1 / 2}$.

These two theorems translate the results [3] for the Euclidean distance to the distance $d(z, w)=|1-\langle z, w\rangle|^{1 / 2}$ in $\partial \mathcal{B}$. It is interesting to remark that in the Euclidean case, the analogue of (1.3) is an equality. On the other hand, these results have a lot of applications [1-3].

The heart of the proof of Theorem A is to reduce it to

Theorem 2. For all non-negative integers $p, q, n(n \geq 1)$ and for all $\beta, 0<\beta<n / 2$, we have, with constants which only depend on $n, \beta$, that

$$
I=\int_{0}^{1}\left(\frac{F(z)}{F(1)}\right)^{2} z^{p+q+\beta-1}(1-z)^{n-2 \beta-1} d z \asymp \frac{\Gamma(p+\beta) \Gamma(q+\beta)}{\Gamma(p+n-\beta) \Gamma(q+n-\beta)}
$$

where $F(z)$ is the hypergeometric function $F(p, q ; p+q+n ; z)$.

The outline of the paper is as follows. In Section 2, we give the proof of Theorem 1. We will prove Theorem 2 in Sections 3 and 4. In Section 5, we will give an open question.

Notation. We denote by $C$ a constant which sometimes can depend on $n$ and $\beta$ and that can change its value from line to line and even in the same line. The expression $A \asymp B$ will mean that there exists a constant $C$, depending at most on $n$ and $\beta$, such that $C^{-1} \leq A / B \leq C$. Finally, $A \sim B$ when $x \rightarrow a$ means that $\lim _{x \rightarrow a} A / B=1$.

\section{Proof of Theorem 1}

Theorem 1. There exists a universal constant $C$, not depending on $n, p, u, m, z$, such that, for all real numbers, $u, p \geq 0, m, n \geq 1,0 \leq z<1$, if we denote

$$
G=F(p+u, m p+1 ;(m+1) p+u+n+1 ; z) B(m p+1, p+u+n)
$$

then

$$
G \geq C L
$$

where

$$
L=t_{0}^{m p+1}\left(1-m\left(1-t_{0}\right)\right)^{p+u}\left(1-t_{0}\right)^{n-1}\left(\frac{1-z}{a^{2}-b^{2} z}\right)^{1 / 4} \frac{1}{m \sqrt{p+1}}
$$

and

$$
\begin{gathered}
t_{0}=\frac{a+b z-\sqrt{(1-z)\left(a^{2}-b^{2} z\right)}}{2 z}=\frac{2}{a+b z+\sqrt{(1-z)\left(a^{2}-b^{2} z\right)}}, \\
a=1+\frac{1}{m}, \quad b=1-\frac{1}{m} .
\end{gathered}
$$

Also,

$$
\lim _{p \rightarrow \infty} \frac{G}{L}=\sqrt{2 \pi} .
$$


In order to prove Theorem 1, we will need the well-known integral expression ([6, p. 99] or [8, p. 20])

$$
G=\int_{0}^{1} t^{m p}(1-t)^{p+u+n-1}(1-z t)^{-p-u} d t .
$$

Accordingly, we can write

$$
G=\int_{0}^{1} e^{p f(t)} g(t) d t
$$

where

$$
f(t)=\log \frac{t^{m}(1-t)}{1-z t} \quad \text { and } \quad g(t)=\frac{(1-t)^{u+n-1}}{(1-z t)^{u}} .
$$

Observe that the function $f$ has a unique maximum $t_{0}$ in $[0,1]$ given by $(2.1)$.

The classical Laplace's method (see e.g., $[7,10]$ ) for asymptotic expansions gives that the principal contribution of the integrand of $G$ is located in a neighborhood of $t_{0}$. Consequently, it will be useful to have some expressions involving $t_{0}$ at our disposal.

Lemma 2.1. If $t_{0}$ is defined by (2.1), we have the formulae

$$
\begin{gathered}
z t_{0}^{2}=(a+b z) t_{0}-1 \\
2 z t_{0}=a+b z-\sqrt{(1-z)\left(a^{2}-b^{2} z\right)} \\
2\left(1-z t_{0}\right)=b(1-z)+\sqrt{(1-z)\left(a^{2}-b^{2} z\right)} \\
=t_{0}\left(a(1-z)+\sqrt{(1-z)\left(a^{2}-b^{2} z\right)}\right), \\
1-t_{0}=\frac{\sqrt{(1-z)\left(a^{2}-b^{2} z\right)}-a(1-z)}{2 z} \\
=\frac{t_{0}}{2}\left(\sqrt{(1-z)\left(a^{2}-b^{2} z\right)}-b(1-z)\right), \\
\left(1-t_{0}\right)\left(1-z t_{0}\right)=\frac{t_{0}}{m}(1-z), \\
\frac{1-t_{0}}{1-z t_{0}}=1-m\left(1-t_{0}\right), \\
f^{\prime \prime}\left(t_{0}\right)=-\frac{m^{2}}{t_{0}^{2}} \sqrt{\frac{a^{2}-b^{2} z}{1-z}} .
\end{gathered}
$$

Proof. In order to find $t_{0}$, we need, of course, to solve the equation $f^{\prime}(t)=0$. This equation is equivalent to (2.2). The identities (2.3)-(2-7) can be obtained by an elementary argument using (2.1) and the definitions of $a$ and $b$. More concretely, (2.6) and (2.7) use (2.2). To obtain (2.8), we use (2.6) and (2.3) in the following way

$$
\begin{aligned}
f^{\prime \prime}\left(t_{0}\right) & =-\frac{m}{t_{0}^{2}}-\frac{1}{\left(1-t_{0}\right)^{2}}+\frac{z^{2}}{\left(1-z t_{0}\right)^{2}} \\
& =-\frac{m^{2}}{t_{0}^{2}} \frac{\frac{1}{m}(1-z)^{2}+\left(1-z t_{0}\right)^{2}-z^{2}\left(1-t_{0}\right)^{2}}{(1-z)^{2}} \\
& =-\frac{m^{2}}{t_{0}^{2}} \frac{a+b z-2 z t_{0}}{1-z} \\
& =-\frac{m^{2}}{t_{0}^{2}} \frac{\sqrt{(1-z)\left(a^{2}-b^{2} z\right)}}{1-z}
\end{aligned}
$$


Proof of Theorem 1. Following Laplace's method (see e.g., $[7,10]$ ), we define a new variable $\tau$ by the equation

$$
f\left(t_{0}\right)-f(t)=\tau^{2}
$$

and the condition that $\tau$ must be an increasing function of $t$.

Using the Taylor's polynomial of degree 2 of $f$ in $t_{0}$, we obtain that if we define $h$ by $t=h(\tau)$, we have

$$
h^{\prime}(0)=\sqrt{\frac{-2}{f^{\prime \prime}\left(t_{0}\right)}} .
$$

Then

$$
G=e^{p f\left(t_{0}\right)} \int_{-\infty}^{\infty} e^{-p \tau^{2}} g(h(\tau)) h^{\prime}(\tau) d \tau
$$

If we use (2.9) and (2.10), then we have that as $p \rightarrow \infty$

$$
G \sim e^{p f\left(t_{0}\right)} g(h(0)) h^{\prime}(0) \int_{-\infty}^{\infty} e^{-p \tau^{2}} d \tau=e^{p f\left(t_{0}\right)} g\left(t_{0}\right) \sqrt{\frac{-2 \pi}{f^{\prime \prime}\left(t_{0}\right) p}} .
$$

Then, using (2.8), we obtain

$$
G \sim\left(\frac{t_{0}^{m}\left(1-t_{0}\right)}{1-z t_{0}}\right)^{p}\left(\frac{1-t_{0}}{1-z t_{0}}\right)^{u}\left(1-t_{0}\right)^{n-1} \sqrt{\frac{2 \pi}{p} \frac{t_{0}^{2}}{m^{2}} \sqrt{\frac{1-z}{a^{2}-b^{2} z}}} .
$$

The identity (2.7) gives

$$
G \sim t_{0}^{m p+1}\left(1-m\left(1-t_{0}\right)\right)^{p+u}\left(1-t_{0}\right)^{n-1} \frac{1}{m} \sqrt{\frac{2 \pi}{p} \sqrt{\frac{1-z}{a^{2}-b^{2} z}}} \sim \sqrt{2 \pi} L .
$$

This proves the last part of Theorem 1. To prove the main part of Theorem 1, we need to estimate $g(h(\tau))$ and $h^{\prime}(\tau)$ near 0 . These estimates must be uniform in $n, p$, $u, m$, and $z$.

For each $0<\varepsilon<1$, we define

$$
\begin{gathered}
t=(1-\varepsilon) t_{0}, \\
x=b+\sqrt{\frac{a^{2}-b^{2} z}{1-z}} \geq 2, \quad \text { if } 0 \leq z<1, \\
w=1+\frac{m \varepsilon}{2} x \geq 1+m \varepsilon, \quad \text { if } 0 \leq z<1 .
\end{gathered}
$$

We need to estimate

$$
\tau^{2}=f\left(t_{0}\right)-f(t)=\log \left(\frac{1}{(1-\varepsilon)^{m}} \frac{1-t_{0}}{1-(1-\varepsilon) t_{0}} \frac{1-(1-\varepsilon) z t_{0}}{1-z t_{0}}\right) .
$$

A computation gives, using (2.4), that

$$
\frac{1-(1-\varepsilon) z t_{0}}{1-z t_{0}}=1-m \varepsilon+\frac{m \varepsilon}{2} x=w-m \varepsilon,
$$

and also, using $(2.5)$, that

$$
\frac{1-t_{0}}{1-(1-\varepsilon) t_{0}}=\frac{1}{1+\frac{m \varepsilon}{2} x}=\frac{1}{w}
$$


where $x, w$ are defined by (2.13) and (2.14). If we substitute (2.16) and (2.17) in (2.15), we obtain

$$
f\left(t_{0}\right)-f(t)=\log \left(\frac{1}{(1-\varepsilon)^{m}}\left(1-\frac{m \varepsilon}{w}\right)\right) \geq \log \frac{1}{(1-\varepsilon)^{m}(1+m \varepsilon)} .
$$

We wish to show that

$$
h^{\prime}(\tau) \geq K h^{\prime}(0) \quad \text { for all } \tau \in\left[\tau_{1}, 0\right],
$$

for some constants $K>0$ and $\tau_{1}<0$ which are independent of $n, p, u, m$ and $z$. In order to obtain this inequality, consider the function $H=h^{-1}$, i.e., $H(t)^{2}=$ $f\left(t_{0}\right)-f(t)$. Then, (2.19) is equivalent to the inequality

$$
\frac{1}{H^{\prime}(t)} \geq \frac{K}{H^{\prime}\left(t_{0}\right)}=K \sqrt{\frac{-2}{f^{\prime \prime}\left(t_{0}\right)}}
$$

for all $t \in\left[t_{1}, t_{0}\right]$, with $t_{1}=h\left(\tau_{1}\right)$. Since we are working with $t<t_{0}$, we have that

$$
H(t)=-\sqrt{f\left(t_{0}\right)-f(t)} .
$$

And recalling (2.8), (2.12), (2.13), and (2.14), we see that to prove (2.20) is equivalent to proving that

$$
\frac{4\left(f\left(t_{0}\right)-f(t)\right)}{f^{\prime}(t)^{2}} \geq 2 K^{2} \frac{t_{0}^{2}}{m^{2}} \frac{1}{x-b}=\frac{2 K^{2} t_{0}^{2}}{m^{2}} \frac{1}{\frac{2}{m \varepsilon}(w-1)-b} .
$$

On the other hand, if $t$ is given by (2.12), computations give, with the help of (2.6), (2.16), and (2.17), that

$$
\begin{aligned}
f^{\prime}(t) & =\frac{m}{(1-\varepsilon) t_{0}}-\frac{1}{1-(1-\varepsilon) t_{0}}+\frac{z}{1-(1-\varepsilon) z t_{0}} \\
& =\frac{m}{(2.16),(2.17)} \frac{1}{(1-\varepsilon) t_{0}}-\frac{1}{w\left(1-t_{0}\right)}+\frac{z}{(w-m \varepsilon)\left(1-z t_{0}\right)} \\
& =\frac{m}{\left.t_{0} .6\right)}\left(\frac{1}{1-\varepsilon}-\frac{w(1-z)-m \varepsilon\left(1-z t_{0}\right)}{w(w-m \varepsilon)(1-z)}\right),
\end{aligned}
$$

and so if we use (2.4) and (2.14) to obtain

$$
1-z t_{0}=\frac{x}{2}(1-z)
$$

then we find that

$$
f^{\prime}(t)=\frac{m}{t_{0}}\left(\frac{1}{1-\varepsilon}-\frac{1}{w(w-m \varepsilon)}\right) .
$$

Substituting (2.18) and (2.22) into the inequality (2.21), we obtain that (2.19) is equivalent to

$$
M(w)=\log \left(\frac{1}{(1-\varepsilon)^{m}}\left(1-\frac{m \varepsilon}{w}\right)\right)-\frac{K^{2}}{\frac{4}{m \varepsilon}(w-1)-2 b}\left(\frac{1}{1-\varepsilon}-\frac{1}{w(w-m \varepsilon)}\right)^{2} \geq 0
$$

for all $w \geq 1+m \varepsilon$ and $\varepsilon \leq \varepsilon_{1}$.

In order to show (2.23), the next lemma plays an important role.

Lemma 2.2. For all $0<\varepsilon<1, m>0, K \leq \sqrt{(1-\varepsilon) / 3}, w \geq 1+m \varepsilon$, we have $M^{\prime}(w)>0$.

In the proof of Lemma 2.2, we will need the next inequality: 
Lemma 2.3. For all $\varepsilon, m>0, w \geq 1+m \varepsilon$, we have

$$
\frac{w(w-m \varepsilon)-(1-\varepsilon)}{w-1-m \varepsilon b / 2} \leq w+2 .
$$

Proof. The restrictions $1+m \varepsilon \leq w$ and $b<1$ give

$$
1+m \varepsilon \leq w+w m \varepsilon(1-b / 2) .
$$

This inequality can be transformed, using the fact that $m=m b+1$, into

$$
1+\varepsilon+m \varepsilon b \leq w+w m \varepsilon-w m \varepsilon b / 2,
$$

which is equivalent to

$$
w(w-m \varepsilon)-(1-\varepsilon) \leq(w+2)(w-1-m \varepsilon b / 2) .
$$

Therefore, we obtain (2.24) by observing that $w-1-m \varepsilon b / 2 \geq m \varepsilon-m \varepsilon b / 2>0$.

Proof of Lemma 2.2. We have that

$$
\begin{aligned}
M^{\prime}(w)=\frac{1}{w-m \varepsilon}- & \frac{1}{w}+\frac{K^{2} m \varepsilon}{4}\left[\frac{1}{(w-1-m \varepsilon b / 2)^{2}}\left(\frac{1}{1-\varepsilon}-\frac{1}{w(w-m \varepsilon)}\right)^{2}\right. \\
& \left.-\frac{2}{w-1-m \varepsilon b / 2}\left(\frac{1}{1-\varepsilon}-\frac{1}{w(w-m \varepsilon)}\right) \frac{2 w-m \varepsilon}{w^{2}(w-m \varepsilon)^{2}}\right] .
\end{aligned}
$$

Then

$$
M^{\prime}(w) \geq \frac{m \varepsilon}{w(w-m \varepsilon)}-\frac{K^{2} m \varepsilon}{2(w-1-m \varepsilon b / 2)}\left(\frac{1}{1-\varepsilon}-\frac{1}{w(w-m \varepsilon)}\right) \frac{2 w-m \varepsilon}{w^{2}(w-m \varepsilon)^{2}} .
$$

We can bound, with the help of (2.24), the term

$$
\begin{aligned}
\frac{1}{w-1-m \varepsilon b / 2}\left(\frac{1}{1-\varepsilon}-\frac{1}{w(w-m \varepsilon)}\right) & =\frac{1}{(1-\varepsilon) w(w-m \varepsilon)} \frac{w(w-m \varepsilon)-(1-\varepsilon)}{w-1-m \varepsilon b / 2} \\
& \leq \frac{w+2}{(1-\varepsilon) w(w-m \varepsilon)} .
\end{aligned}
$$

We also can obtain an upper bound of the term

$$
\frac{2 w-m \varepsilon}{w^{2}(w-m \varepsilon)^{2}}<\frac{2 w}{w^{2}}=\frac{2}{w} .
$$

Substituting (2.26) and (2.27) into (2.25), we obtain

$$
\begin{aligned}
M^{\prime}(w) & >\frac{m \varepsilon}{w(w-m \varepsilon)}-\frac{K^{2} m \varepsilon}{w} \frac{w+2}{(1-\varepsilon) w(w-m \varepsilon)} \\
& =\frac{m \varepsilon}{w(w-m \varepsilon)}\left(1-\frac{K^{2}}{1-\varepsilon}\left(1+\frac{2}{w}\right)\right) .
\end{aligned}
$$

The hypothesis on $K$ in Lemma 2.2 gives that $K^{2} \leq(1-\varepsilon) / 3$, and then

$$
\frac{K^{2}}{1-\varepsilon}\left(1+\frac{2}{w}\right) \leq 1 \text {. }
$$

This implies $M^{\prime}(w)>0$. 
Consequently, if $K \leq \sqrt{(1-\varepsilon) / 3}$, we have that

$$
M(w) \geq M(1+m \varepsilon),
$$

and so, we only need to prove that $N(\varepsilon)=M(1+m \varepsilon) \geq 0$.

Lemma 2.4. For all $0<\varepsilon<1, m>0, K \leq 1-\varepsilon$, we have that $N(\varepsilon) \geq N(0)=0$. Proof. It is enough to show that $N^{\prime}(\varepsilon)>0$. Recall that

$$
N(\varepsilon)=M(1+m \varepsilon)=\log \left(\frac{1}{(1-\varepsilon)^{m}(1+m \varepsilon)}\right)-\frac{K^{2}}{2 a}\left(\frac{1}{1-\varepsilon}-\frac{1}{1+m \varepsilon}\right)^{2} .
$$

Therefore,

$$
N^{\prime}(\varepsilon)=\frac{m}{1-\varepsilon}-\frac{m}{1+m \varepsilon}-\frac{K^{2}}{a}\left(\frac{1}{1-\varepsilon}-\frac{1}{1+m \varepsilon}\right)\left(\frac{1}{(1-\varepsilon)^{2}}+\frac{m}{(1+m \varepsilon)^{2}}\right) .
$$

Using the fact that

$$
\frac{1}{1-\varepsilon}-\frac{1}{1+m \varepsilon}=\frac{m \varepsilon a}{(1-\varepsilon)(1+m \varepsilon)}
$$

we have

$$
N^{\prime}(\varepsilon)=\frac{m \varepsilon}{(1-\varepsilon)(1+m \varepsilon)}\left(m a-K^{2}\left(\frac{1}{(1-\varepsilon)^{2}}+\frac{m}{(1+m \varepsilon)^{2}}\right)\right) .
$$

The hypothesis $K^{2} \leq(1-\varepsilon)^{2}$ gives

$$
\frac{K^{2}}{(1-\varepsilon)^{2}}\left(1+m \frac{(1-\varepsilon)^{2}}{(1+m \varepsilon)^{2}}\right)<1+m=m a
$$

and this implies $N^{\prime}(\varepsilon)>0$.

It is convenient to make a back-up of our results. We have showed that if $0<\varepsilon<1$, $m>0, K \leq \min \{\sqrt{(1-\varepsilon) / 3}, 1-\varepsilon\}$, then for $t=(1-\varepsilon) t_{0}$

$$
\frac{1}{H^{\prime}(t)} \geq \frac{K}{H^{\prime}\left(t_{0}\right)} \text {. }
$$

Take $0<\varepsilon \leq \varepsilon_{0}<1$ and $K=\min \left\{\sqrt{\left(1-\varepsilon_{0}\right) / 3}, 1-\varepsilon_{0}\right\} \leq \min \{\sqrt{(1-\varepsilon) / 3}, 1-\varepsilon\}$, then we have

$$
h^{\prime}(H(t)) \geq K h^{\prime}(0) \quad \text { for all } t \in\left[\left(1-\varepsilon_{0}\right) t_{0}, t_{0}\right] .
$$

Then (2.18) gives, if $m \geq 1$,

$$
H\left(\left(1-\varepsilon_{0}\right) t_{0}\right)^{2}=f\left(t_{0}\right)-f\left(\left(1-\varepsilon_{0}\right) t_{0}\right) \geq \log \frac{1}{\left(1-\varepsilon_{0}\right)^{m}\left(1+m \varepsilon_{0}\right)} \geq \log \frac{1}{1-\varepsilon_{0}^{2}} \equiv \tau_{1}^{2}
$$

where the last inequality is true since $m \geq 1$. Of course, $H\left(\left(1-\varepsilon_{0}\right) t_{0}\right)$ and $\tau_{1}$ are negative numbers and we have

$$
H\left(\left(1-\varepsilon_{0}\right) t_{0}\right) \leq \tau_{1}
$$

and then

$$
h^{\prime}(\tau) \geq K h^{\prime}(0) \quad \text { for all } t \in\left[\tau_{1}, 0\right]
$$


Therefore, (2.11) and the positivity of the integrand give that

$$
\begin{aligned}
G & \geq e^{p f\left(t_{0}\right)} \int_{\tau_{1}}^{0} e^{-p \tau^{2}} g(h(\tau)) h^{\prime}(\tau) d \tau \\
& \geq K h^{\prime}(0) e^{p f\left(t_{0}\right)} \int_{\tau_{1}}^{0} e^{-p \tau^{2}} g(h(\tau)) d \tau .
\end{aligned}
$$

Observe that $h(\tau)$ is an increasing function on $\tau$ and $g(t)$ is a decreasing function on $t$ (because $n \geq 1$ ). Then

$$
G \geq K e^{p f\left(t_{0}\right)} g\left(t_{0}\right) h^{\prime}(0) \int_{\tau_{1}}^{0} e^{-p \tau^{2}} d \tau \geq \frac{C}{\sqrt{p+1}} e^{p f\left(t_{0}\right)} g\left(t_{0}\right) h^{\prime}(0) .
$$

By observing that

$$
\lim _{p \rightarrow \infty} \frac{\int_{\tau_{1}}^{0} e^{-p \tau^{2}} d \tau}{\int_{-\infty}^{\infty} e^{-p \tau^{2}} d \tau}=\frac{1}{2}
$$

this completes the proof of Theorem 1 .

\section{Proof of first part of Theorem 2}

In this section, we will prove one half of Theorem 2. More concretely:

Theorem 2.1. There exists a positive constant $C$, depending only on $n$ and $\beta$, such that for all nonnegative integers $p, q, n(n \geq 1)$ and for all $\beta, 0<\beta<n / 2$, we have

$$
I=\int_{0}^{1}\left(\frac{F(z)}{F(1)}\right)^{2} z^{p+q+\beta-1}(1-z)^{n-2 \beta-1} d z \geq C \frac{\Gamma(p+\beta) \Gamma(q+\beta)}{\Gamma(p+n-\beta) \Gamma(q+n-\beta)}
$$

where $F(z)$ is the hypergeometric function $F(p, q ; p+q+n ; z)$.

If $p$ or $q$ is 0 , then $F$ is the constant 1 , and $I$ is the Beta function $B(p+q+\beta, n-2 \beta)$. Thus we can assume that $p$ and $q$ are not zero. By the symmetry of the hypergeometric function in the two first variables, it is enough to prove the inequality for $p \geq q$. Let $p=m q$, with $m \geq 1$.

The corollary following Theorem 1 gives that

$$
F(z) B(m q, q+n) \geq C L .
$$

The Gauss summation formula ([6, p. 99], [8, p. 28]) gives

$$
F(1)=\frac{\Gamma(m q+q+n) \Gamma(n)}{\Gamma(m q+n) \Gamma(q+n)}
$$

and, therefore,

$$
F(z) B(m q, q+n)=\frac{F(z)}{F(1)} \frac{\Gamma(m q) \Gamma(n)}{\Gamma(m q+n)} \leq \frac{F(z)}{F(1)} \frac{C}{(m q)^{n}}
$$

where we have used the well-known fact:

Proposition 3.1. For all fixed real numbers $u, v$, we have that

$$
\frac{\Gamma(x+u)}{\Gamma(x+v)} \sim \frac{1}{x^{v-u}} \quad \text { when } x \rightarrow+\infty
$$


Hence,

$$
\frac{F(z)}{F(1)} \geq C(n) m^{n-1} q^{n-1 / 2} t_{0}^{m q}\left(1-m\left(1-t_{0}\right)\right)^{q}\left(1-t_{0}\right)^{n-1}\left(\frac{1-z}{a^{2}-b^{2} z}\right)^{1 / 4}
$$

where $a=1+1 / m$ and $b=1-1 / m$. Then we have

$$
I \geq C m^{2 n-2} q^{2 n-1} J
$$

where

$$
J=\int_{0}^{1} e^{q f(z)} g(z) d z
$$

and

$$
\begin{gathered}
f(z)=\log \left(t_{0}^{2 m}\left(1-m\left(1-t_{0}\right)\right)^{2} z^{m+1}\right), \\
g(z)=\frac{\left(1-t_{0}\right)^{2 n-2}}{\sqrt{a^{2}-b^{2} z}} z^{\beta-1}(1-z)^{n-2 \beta-1 / 2} .
\end{gathered}
$$

The functions $t_{0}$ and $f$ are increasing; observe that

$$
\frac{d}{d z} \frac{2}{t_{0}}=b-\frac{b^{2}(1-z)+2 / m}{\sqrt{(1-z)\left(a^{2}-b^{2} z\right)}},
$$

and that this function is negative because

$$
b \sqrt{(1-z)\left(a^{2}-b^{2} z\right)} \leq 2 \frac{1}{\sqrt{m}} b \sqrt{1-z} \leq \frac{1}{m}+b^{2}(1-z) .
$$

Following Laplace's method [7, 10], we introduce the new variable $\tau=-f(z)$; then, if $z=h(\tau)$, we have

$$
J=\int_{0}^{\infty} e^{-q \tau} g(h(\tau))\left|h^{\prime}(\tau)\right| d \tau .
$$

In order to bound $J$, we need some estimates for the function

$$
r(z)=\frac{2 / m}{\sqrt{4 / m+b^{2}(1-z)}+a \sqrt{1-z}} .
$$

The function $r$ is increasing for $0 \leq z \leq 1$; then we have that $1 /(m+1) \leq r(z) \leq 1 / \sqrt{m}$. For each $k$ such that $\sqrt{m} /(m+1) \leq k \leq 1$, there is a unique $0 \leq z_{m} \leq 1$ such that $r\left(z_{m}\right)=k / \sqrt{m}$. A computation shows that

$$
\sqrt{1-z_{m}}=\frac{1}{2 k}\left(a \sqrt{m}-\sqrt{b^{2} m+4 k^{2}}\right)
$$

and

$$
z_{m}=\frac{1}{4 k^{2}}\left(2 a \sqrt{b^{2} m^{2}+4 k^{2} m}-m\left(a^{2}+b^{2}\right)\right) .
$$

We need the following lemma in order to prove that there is an interval $[0, A]$ for the variable $\tau$, for some universal constant $A$, in which the estimates are valid.

In what follows, we choose $k=(\sqrt{65}-1) / 8$ and $z_{m}$ such that $r\left(z_{m}\right)=k / \sqrt{m}$ for this particular $k$.

Lemma 3.1. If $\tau_{m}$ is defined as $\tau_{m}=-f\left(z_{m}\right)$, then there is a universal positive constant $A$ such that $\tau_{m} \geq A$ for all $m \geq 1$.

In order to prove this result, we need some inequalities. 
Lemma 3.2. For all $z \in\left[z_{m}, 1\right]$ and for all $m \geq 1$, we have that

$$
\begin{gathered}
k \sqrt{\frac{1-z}{m}} \leq 1-t_{0} \leq \sqrt{\frac{1-z}{m}}, \\
1-\sqrt{m(1-z)} \leq 1-m\left(1-t_{0}\right) \leq 1-k \sqrt{m(1-z)}, \\
1-t_{0} \leq 1-t_{0}\left(z_{m}\right)<2 \frac{1-k^{2}}{m}<\frac{2}{m}, \\
\sqrt{m(1-z)} \leq \sqrt{m\left(1-z_{m}\right)}<2 \frac{1-k^{2}}{k}=\frac{1}{2}, \\
z_{m} \in\left[\frac{3}{4}, 1\right], \\
k \sqrt{\frac{1-z}{m}} \leq-\log t_{0} \leq 2 \sqrt{\frac{1-z}{m}}, \\
0 \leq-\log z \leq \sqrt{\frac{1-z}{m}}, \\
a^{2}-b^{2} z \leq \frac{5}{m}, \\
4 k \sqrt{m(1-z)} \leq \tau \leq 10 \sqrt{m(1-z)} .
\end{gathered}
$$

Proof. A straightforward computation using (2.5) shows that

$$
1-t_{0}=r(z) \sqrt{1-z}
$$

This proves (3.2a) and (3.2b), since $r$ is an increasing function for $0 \leq z \leq 1$.

Since $t_{0}=t_{0}(z)$ is an increasing function of $z$, we have, using the fact that $r\left(z_{m}\right)=$ $k / \sqrt{m}$ and also (3.1), that

$$
1-t_{0} \leq 1-t_{0}\left(z_{m}\right)=r\left(z_{m}\right) \sqrt{1-z_{m}}=k \sqrt{\frac{1-z_{m}}{m}}=\frac{1}{2}\left(a-\sqrt{b^{2}+\frac{4 k^{2}}{m}}\right)
$$

so

$$
1-t_{0} \leq 1-t_{0}\left(z_{m}\right)=\frac{1}{2} \frac{\frac{4}{m}-\frac{4 k^{2}}{m}}{a+\sqrt{b^{2}+\frac{4 k^{2}}{m}}}<2 \frac{1-k^{2}}{m}
$$

which proves $(3.2 \mathrm{c})$.

In order to prove (3.2d), it is enough to observe that (3.3) and (3.2c) give

$$
\sqrt{m\left(1-z_{m}\right)}=\frac{m}{k}\left(1-t_{0}\left(z_{m}\right)\right)<2 \frac{1-k^{2}}{k}
$$

and this last number is equal to $1 / 2$ because of our choice of the constant $k$.

(3.2e) follows directly from $(3.2 \mathrm{~d})$.

(3.2a) gives

$$
1-\sqrt{\frac{1-z}{m}} \leq t_{0} \leq 1-k \sqrt{\frac{1-z}{m}} .
$$

If we use the inequalities

$$
x \leq-\log (1-x) \leq \frac{x}{1-x} \quad \text { for all } x \in(0,1),
$$

and we observe (see $(3.2 \mathrm{~d}))$ that $\sqrt{(1-z) / m} \leq 1 / 2$, then we obtain (3.2f).

$(3.2 \mathrm{~g})$ can be deduced like (3.2f) using $(3.2 \mathrm{~b})$ instead of $(3.2 \mathrm{a})$. 
The inequality $(3.2 \mathrm{~h})$ follows from

$$
\begin{aligned}
-\log z & \leq \frac{1-z}{z} \leq \frac{4}{3}(1-z) \\
& =\frac{4}{3} \sqrt{m(1-z)} \sqrt{\frac{1-z}{m}} \leq \sqrt{\frac{1-z}{m}}
\end{aligned}
$$

where we have used (3.2d) and (3.2e).

(3.2i) can be proved using $(3.2 \mathrm{~d})$ in the following way

$$
a^{2}-b^{2} z=a^{2}-b^{2}+b^{2}(1-z) \leq \frac{4}{m}+1-z_{m} \leq \frac{5}{m} .
$$

Finally, (3.2j) follows from (3.2f), (3.2g), and (3.2h).

Proof of Lemma 3.1. The inequality (3.2j) with $z=z_{m}$ gives

$$
\tau_{m} \geq 4 k \sqrt{m\left(1-z_{m}\right)} .
$$

On the other hand, (3.1) allows us to compute

$$
\begin{aligned}
\lim _{m \rightarrow \infty} \sqrt{m\left(1-z_{m}\right)} & =\lim _{m \rightarrow \infty} \frac{m}{2 k}\left(a-\sqrt{b^{2}+\frac{4 k^{2}}{m}}\right) \\
& =\lim _{m \rightarrow \infty} \frac{m}{2 k} \frac{\frac{4}{m}-\frac{4 k^{2}}{m}}{a+\sqrt{b^{2}+\frac{4 k^{2}}{m}}}=\frac{1-k^{2}}{k}=\frac{1}{4}
\end{aligned}
$$

where the last equality is true because of our choice of $k$.

Since $\tau_{m}>0$ for all $m \geq 1$ and $\liminf _{m \rightarrow \infty} \tau_{m} \geq k$, we have that

$$
A=\inf _{m} \tau_{m}>0 \text {. }
$$

Lemma 3.3. If $z \in\left[z_{m}, 1\right]$, then the derivative with respect to $z$ of the function $t_{0}$ satisfies

$$
t_{0}^{\prime}(z) \leq \frac{2}{\sqrt{m(1-z)}}
$$

Proof. Recall (see (2.1)) that

$$
t_{0}(z)=\frac{a}{2 z}+\frac{b}{2}-\frac{\sqrt{a^{2}-b^{2} z}}{2 z} \sqrt{1-z} .
$$

Therefore,

$$
t_{0}^{\prime}(z)=\frac{-a}{2 z^{2}}+\frac{2 a^{2}-b^{2} z}{4 z^{2} \sqrt{a^{2}-b^{2} z}} \sqrt{1-z}+\frac{\sqrt{a^{2}-b^{2} z}}{2 z} \frac{1}{2 \sqrt{1-z}} .
$$

Hence,

$$
t_{0}^{\prime}(z) \leq \frac{a^{2}}{2 z^{2} \sqrt{a^{2}-b^{2} z}} \sqrt{1-z}+\frac{1}{\sqrt{m}} \frac{1}{\sqrt{1-z}}
$$

where we have used (3.2e) and (3.2i). On the other hand, using $a^{2}-b^{2}=4 / m$ and (3.2e), we have that

$$
\frac{a^{2}}{2 z^{2} \sqrt{a^{2}-b^{2} z}} \sqrt{1-z} \leq \frac{a^{2}}{2 z^{2} \sqrt{a^{2}-b^{2}}} \sqrt{1-z} \leq 2 \sqrt{m(1-z)} .
$$


Besides, using (3.2d), we deduce

$$
2 \sqrt{m(1-z)} \leq \frac{1}{\sqrt{m(1-z)}} .
$$

Finally, substituting these two last inequalities in (3.5), we obtain (3.4).

Lemma 3.4. For all $z \in\left[z_{m}, 1\right]$, we have that

$$
f^{\prime}(z) \leq C \sqrt{\frac{m}{1-z}} .
$$

Proof. Recall that

$$
f(z)=\log \left(t_{0}^{2 m}\left(1-m\left(1-t_{0}\right)\right)^{2} z^{m+1}\right) .
$$

Hence,

$$
f^{\prime}(z)=2 m \frac{t_{0}^{\prime}}{t_{0}}+2 m \frac{t_{0}^{\prime}}{1-m\left(1-t_{0}\right)}+\frac{m+1}{z} .
$$

Using (3.2a) and $(3.2 \mathrm{~d})$, we have that $t_{0} \geq 1 / 2$. Similarly, using $(3.2 \mathrm{~b})$ and $(3.2 \mathrm{~d})$ again, one deduces that $1-m\left(1-t_{0}\right) \geq 1 / 2$. Therefore, recalling (3.2e) and (3.4), we obtain that

$$
f^{\prime}(z) \leq 4 m\left(t_{0}^{\prime}+t_{0}^{\prime}+1\right) \leq C \sqrt{\frac{m}{1-z}}
$$

Lemma 3.5. Let $A=\inf _{m} \tau_{m}$. Then, for all $\tau \in[0, A]$, we have that

$$
\begin{gathered}
\left|h^{\prime}(\tau)\right| \geq \frac{C \tau}{m}, \\
g(h(\tau)) \geq \frac{C \tau^{4 n-4 \beta-3}}{m^{3 n-2 \beta-3}} .
\end{gathered}
$$

Proof. First, recalling that $h=(-f)^{-1}$ and using (3.6) and (3.2j), we have that

$$
\left|h^{\prime}(\tau)\right|=\frac{1}{f^{\prime}(z)} \geq C \sqrt{\frac{1-z}{m}} \geq \frac{C \tau}{m} .
$$

This proves (3.7). Secondly, recall also that

$$
g(h(\tau))=g(z)=\frac{\left(1-t_{0}\right)^{2 n-2}}{\sqrt{a^{2}-b^{2} z}} z^{\beta-1}(1-z)^{n-2 \beta-1 / 2} .
$$

Therefore, using (3.2a), (3.2i), and (3.2e), we have that

$$
g(h(\tau)) \geq C\left(\frac{1-z}{m}\right)^{n-1} \sqrt{m}(1-z)^{n-2 \beta-1 / 2}=C \frac{(1-z)^{2 n-2 \beta-3 / 2}}{m^{n-3 / 2}}
$$

so, $(3.2 \mathrm{j})$ gives the result.

Proof of Theorem 2.1. Recall that we need a lower bound of the integral

$$
J=\int_{0}^{\infty} e^{-q \tau} g(h(\tau))\left|h^{\prime}(\tau)\right| d \tau .
$$


Using Lemma 3.5 and the positivity of the integrand, we have that

$$
\begin{aligned}
J & \geq \int_{0}^{A} e^{-q \tau} g(h(\tau))\left|h^{\prime}(\tau)\right| d \tau \\
& \geq \frac{C}{m^{3 n-2 \beta-2}} \int_{0}^{A} e^{-q \tau} \tau^{4 n-4 \beta-2} d \tau \\
& \geq \frac{C}{m^{3 n-2 \beta-2}} \frac{\Gamma(4 n-4 \beta-1)}{q^{4 n-4 \beta-1}}
\end{aligned}
$$

where we have used the elementary fact that

$$
\lim _{q \rightarrow \infty} \frac{\int_{0}^{A} e^{-q \tau} \tau^{4 n-4 \beta-2} d \tau}{\int_{0}^{\infty} e^{-q \tau} \tau^{4 n-4 \beta-2} d \tau}=1
$$

Recalling that $I \geq C m^{2 n-2} q^{2 n-1} J$ and using (3.9), we obtain that

$$
I \geq C \frac{1}{m^{n-2 \beta}} \frac{1}{q^{2 n-4 \beta}}=\frac{C}{(p q)^{n-2 \beta}} .
$$

Finally, (3.10) and Proposition 3.1 give Theorem 2.1.

\section{Proof of second part of Theorem 2}

To finish the proof of Theorem 2, we need only to prove the reverse inequality.

Theorem 2.2. There exists a positive constant $C$, depending only on $n$ and $\beta$, such that for all nonnegative integers $p, q, n(n \geq 1)$ and for all $\beta, 0<\beta<n / 2$, we have

$$
I=\int_{0}^{1}\left(\frac{F(z)}{F(1)}\right)^{2} z^{p+q+\beta-1}(1-z)^{n-2 \beta-1} d z \leq C \frac{\Gamma(p+\beta) \Gamma(q+\beta)}{\Gamma(p+n-\beta) \Gamma(q+n-\beta)} .
$$

In order to prove Theorem 2.2, we will need some lemmas.

Lemma 4.1. For $p, q, n, z$ as in Theorem 2, we have

$$
(B(q, p+n) F(p, q ; p+q+n ; z))^{2} \leq B(q, n) B(q, 2 p+n) F(2 p, q ; 2 p+q+n ; z) .
$$

Proof. We have ([6, p. 99], [8, p. 20]) that

$$
\begin{aligned}
B(q, p+n) & F(p, q ; p+q+n ; z)=\int_{0}^{1} t^{q-1}(1-t)^{p+n-1}(1-z t)^{-p} d t \\
& =\int_{0}^{1} t^{(q-1) / 2}(1-t)^{(n-1) / 2} t^{(q-1) / 2}(1-t)^{p+(n-1) / 2}(1-z t)^{-p} d t
\end{aligned}
$$

so, using the Cauchy-Schwarz inequality,

$$
\begin{aligned}
(B(q, p+n) F(p, q ; p+q+n ; z))^{2} \leq & \left(\int_{0}^{1} t^{q-1}(1-t)^{n-1} d t\right) \\
& \times\left(\int_{0}^{1} t^{q-1}(1-t)^{2 p+n-1}(1-z t)^{-2 p} d t\right) \\
= & B(q, n) B(q, 2 p+n) F(2 p, q ; 2 p+q+n ; z) .
\end{aligned}
$$

Let ${ }_{3} F_{2}(a, b, c ; d, e ; z)$ denote the generalized hypergeometric function

$$
{ }_{3} F_{2}(a, b, c ; d, e ; z)=\sum_{k=0}^{\infty} \frac{(a)_{k}(b)_{k}(c)_{k}}{(d)_{k}(e)_{k}} \frac{z^{k}}{k !} .
$$

We have 
Lemma 4.2. There exist constants $C_{1}, C_{2}$ depending only on $n$ and $\beta$ such that

$$
C_{1} \leq \frac{{ }_{3} F_{2}(2 p, q, p+q+\beta ; 2 p+q+n, p+q+n-\beta ; z)}{F(2 p, q ; 2 p+q+2 n-2 \beta ; z)} \leq C_{2} .
$$

Proof. By comparing the $k$-th terms of each series, we have that

$$
\begin{aligned}
Q_{k} & \equiv \frac{\frac{(2 p)_{k}(q)_{k}(p+q+\beta)_{k}}{(2 p+q+n)_{k}(p+q+n-\beta)_{k}} \frac{z^{k}}{k !}}{\frac{(2 p)_{k}(q)_{k}}{(2 p+q+2 n-2 \beta)_{k}} \frac{z^{k}}{k !}} \\
& =\frac{(2 p+q+2 n-2 \beta)_{k}}{(2 p+q+n)_{k}} \frac{(p+q+\beta)_{k}}{(p+q+n-\beta)_{k}} \\
& =\frac{\Gamma(2 p+q+2 n-2 \beta+k) \Gamma(p+q+\beta+k)}{\Gamma(2 p+q+n+k) \Gamma(p+q+n-\beta+k)} \frac{\Gamma(2 p+q+n) \Gamma(p+q+n-\beta)}{\Gamma(2 p+q+2 n-2 \beta) \Gamma(p+q+\beta)} .
\end{aligned}
$$

If we denote

$$
A(p, q) \equiv \frac{\Gamma(2 p+q+n) \Gamma(p+q+n-\beta)}{\Gamma(2 p+q+2 n-2 \beta) \Gamma(p+q+\beta)}
$$

again using Proposition 3.1, then we have that

$$
A(p, q) \sim \frac{(p+q)^{n-2 \beta}}{(2 p+q)^{n-2 \beta}}, \quad \text { if } p+q \rightarrow \infty
$$

so there exists a constant $C=C(n, \beta)$ such that

$$
C^{-1} \leq A(p, q) \leq C \quad \text { for all } p, q \geq 0
$$

Also,

$$
C^{-1} \leq A(p, q+k) \leq C \quad \text { for all } p, q, k \geq 0
$$

Therefore,

$$
C^{-2} \leq Q_{k}=\frac{A(p, q)}{A(p, q+k)} \leq C^{2} \quad \text { for all } k \geq 0
$$

and this implies the lemma.

Proof of Theorem 2.2. The Gauss summation formula ([6, p. 99], [8, p. 28]) gives

$$
F(1)=\frac{\Gamma(p+q+n) \Gamma(n)}{\Gamma(p+n) \Gamma(q+n)}=\frac{B(q, n)}{B(q, p+n)}
$$


therefore,

$$
\begin{aligned}
& I=\frac{1}{B(q, n)^{2}} \int_{0}^{1}(F(z) B(q, p+n))^{2} z^{p+q+\beta-1}(1-z)^{n-2 \beta-1} d z \\
& \underset{\text { Lemma }}{\leq} \frac{B(q, 2 p+n)}{B(q, n)} \int_{0}^{1} F(2 p, q ; 2 p+q+n ; z) z^{p+q+\beta-1}(1-z)^{n-2 \beta-1} d z \\
& =\frac{B(q, 2 p+n)}{B(q, n)} \int_{0}^{1} \sum_{k=0}^{\infty} \frac{(2 p)_{k}(q)_{k}}{(2 p+q+n)_{k} k !} z^{k+p+q+\beta-1}(1-z)^{n-2 \beta-1} d z \\
& =\frac{B(q, 2 p+n)}{B(q, n)} \sum_{k=0}^{\infty} \frac{(2 p)_{k}(q)_{k}}{(2 p+q+n)_{k} k !} \frac{\Gamma(k+p+q+\beta) \Gamma(n-2 \beta)}{\Gamma(k+p+q+n-\beta)} \\
& =\frac{B(q, 2 p+n)}{B(q, n)} B(p+q+\beta, n-2 \beta) \\
& \times{ }_{3} F_{2}(2 p, q, p+q+\beta ; 2 p+q+n, p+q+n-\beta ; 1) \\
& \underset{\text { Lemma } 4.2}{\leq} C \frac{\Gamma(2 p+n) \Gamma(q+n)}{\Gamma(2 p+q+n)} \frac{\Gamma(p+q+\beta)}{\Gamma(p+q+n-\beta)} F(2 p, q ; 2 p+q+2 n-2 \beta ; 1) \\
& =C \frac{\Gamma(2 p+n) \Gamma(q+n) \Gamma(p+q+\beta)}{\Gamma(2 p+q+n) \Gamma(p+q+n-\beta)} \frac{\Gamma(2 p+q+2 n-2 \beta)}{\Gamma(2 p+2 n-2 \beta) \Gamma(q+2 n-2 \beta)} \\
& \leq C \frac{(2 p+q+1)^{n-2 \beta}}{(2 p+1)^{n-2 \beta}(q+1)^{n-2 \beta}(p+q+1)^{n-2 \beta}} \\
& \leq \frac{C}{(p+1)^{n-2 \beta}(q+1)^{n-2 \beta}} \\
& \leq C \frac{\Gamma(p+\beta) \Gamma(q+\beta)}{\Gamma(p+n-\beta) \Gamma(q+n-\beta)}
\end{aligned}
$$

where again we have used the Gauss summation formula and used Proposition 3.1 twice.

\section{An open question}

In this section, we formulate an open question which refers to estimates of the square of an hypergeometric function:

Is it true that

$$
(F(p, q ; p+q+n ; z))^{2} \asymp F(2 p, 2 q ; 2 p+2 q+2 n-1 / 2 ; z)
$$

for $p, q, n$ positive integers, $0 \leq z \leq 1$ ?

We know three cases in which this is true: if $n=1 / 2$ (though $1 / 2$ is not an integer!) as a consequence of the Clausen formula, see e.g., [8, p. 75]; if $z=1$ (using the Gauss summation formula) or $z=0$; and if $p$ or $q$ is zero. On the other hand, we have a formal argument based on the asymptotic behaviour of the hypergeometric function stated in Theorem 1, which would give a positive answer to the question above.

If the answer to this question is yes, then this would simplify considerably the proof of Theorem 2 by using the ideas contained in the proof of Theorem 2.2.

Acknowledgements. We would like to thank R. Askey and D. Zeilberger for some helpful communications and José L. Fernández for many useful discussions. We would like also to thank the referees for their careful reading of the manuscript and their suggestions. Research of the second author was supported by a grant of the CICYT, Ministerio de Educación y Ciencia, Spain. 


\section{References}

1. J. L. Fernández and D. Pestana, Distortion of boundary sets under inner functions and applications, Indiana J. Math. 41 (1992), 439-448.

2. - Radial images by holomorphic mappings, To appear in Proc. Amer. Math. Soc.

3. J. L. Fernández, D. Pestana, and J. M. Rodríguez, Distortion of boundary sets under inner functions (II), To appear in Pacific J. Math.

4. D. Pestana and J. M. Rodríguez, Capacity distortion by inner functions in the unit ball of $\mathbf{C}^{n}$, Preprint, 1995.

5. G. B. Folland, Spherical harmonic expansion of the Poisson-Szegö kernel for the ball, Proc. Amer. Math. Soc. 47 (1975), 401-408.

6. Y. L. Luke, The Special Functions and Their Approximations, Academic Press, New York, 1969.

7. F. W. J. Olver, Asymptotics and Special Functions, Academic Press, New York, 1974.

8. L. J. Slater, Generalized Hypergeometric Functions, Cambridge University Press, Cambridge, 1966.

9. G. N. Watson, Asymptotic expansions of hypergeometric functions, Trans. Cambridge Phil. Soc. 22 (1918), 277-308.

10. R. Wong, Asymptotic Approximation of Integrals, Academic Press, New York, 1989.

Universidad Carlos III de Madrid, Butarque, 15, Leganés, 28911 Madrid, SPAIN

E-mail: domingo@dulcinea.uc3m.es, rodrig@dulcinea.uc3m.es 